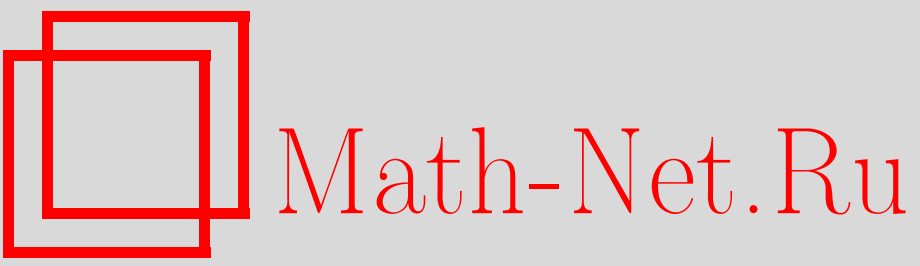

Ю. П. Чубурин, Возмущение резонансов и собственных значений на непрерывном спектре оператора Шредингера для кристаллической пленки, ТМФ, 2005, том 143, номер 3, 417-430

DOI: https://doi.org/10.4213/tmf1822

Использование Общероссийского математического портала Math-Net.Ru подразумевает, что вы прочитали и согласны с пользовательским соглашением

http: //www . mathnet.ru/rus/agreement

Параметры загрузки:

IP : 54.237 .59 .107

26 апреля 2023 г., 16:34:46 
ТЕОРЕТИЧЕСКАЯ

И МАТЕМАТИЧЕСКАЯ

ФИЗИКА

Том 143, № 3

июнь, 2005

(C) 2005 г.

Ю. П. Чубурин*

\section{ВОЗМУЩЕНИЕ РЕЗОНАНСОВ И СОБСТВЕННЫХ ЗНАЧЕНИЙ НА НЕПРЕРЫВНОМ СПЕКТРЕ ОПЕРАТОРА ШРЕДИНГЕРА ДЛЯ КРИСТАЛЛИЧЕСКОЙ ПЛЕНКИ}

Для резонансов и собственных значений на непрерывном спектре получены формулы, подобные формулам обычной теории возмущений. Доказано, что хотя мнимая часть поправки первого поря дка собственного значения на непрерывном спектре равна нулю, но возмущенная собственная функция, как правило, перестает быть квадратично суммируемой.

Ключевые слова: оператор Шредингера, теория возмущений, резонанс, собственное значение.

\section{1. ВВЕДЕНИЕ}

Рассмотрим оператор Шредингера $H=H_{0}+V(x), x \in \mathbb{R}^{3}$, с вешественным потенциалом $V(x) \neq 0$, периодическим по переменным $x_{1}, x_{2}$ с периодом единица и удовлетворяющим оценке

$$
|V(x)| \leqslant C e^{-a\left|x_{3}\right|}
$$

где $a>0$. Оператор $H$ разлагается в прямом интеграле пространств $\int_{\Omega^{*}}^{\oplus} L^{2}(\Omega) d k_{\|}$по операторам $H\left(k_{\|}\right)=-\Delta+V(x)$, определенными на блоховских по переменным $x_{1}, x_{2}$ функциях (см. [1], [2]). Здесь $\Omega=[0,1)^{2} \times \mathbb{R}$ и $\Omega^{*}=[-\pi, \pi)^{2}$ - ячейки в прямой и обратной решетках, $k_{\|} \in \Omega^{*}$ - квазиимпульс, блоховские функции - это сужения на $\Omega$ функций $\psi$, определенных на $\mathbb{R}^{3}$ и удовлетворяюших соотношениям

$$
\psi\left(x+\left(n_{\|}, 0\right)\right)=e^{i\left(k_{\|}, n_{\|}\right)} \psi(x), \quad n_{\|} \in \mathbb{Z}^{2} .
$$

Сушественный спектр оператора $H\left(k_{\|}\right)$совпадает с $\left[k_{\|}^{2}, \infty\right)$, т.е. со спектром оператора $H_{0}\left(k_{\|}\right)[3]$. Обозначим через $R_{0}\left(k_{\|}, E\right)=\left(H_{0}\left(k_{\|}\right)-E\right)^{-1}$ резольвенту оператора

* Физико-технический институт УрО РАН, Ижевск, Россия. E-mail: chuburin@otf.pti.udm.ru 
$H_{0}\left(k_{\|}\right)=-\Delta$, а через $R\left(k_{\|}, E\right)=\left(H\left(k_{\|}\right)-E\right)^{-1}$ - резольвенту оператора $H\left(k_{\|}\right)$. Ядро резольвенты $R_{0}\left(k_{\|}, E\right)$ имеет вид [4]

$$
\begin{aligned}
& G_{0}\left(x-y, k_{\|}, E\right)= \\
& \quad=-\sum_{n_{\|} \in \mathbb{Z}^{2}} \frac{\exp \left\{i\left(\left(k_{\|}+2 \pi n_{\|}, \sqrt{E-\left(k_{\|}+2 \pi n_{\|}\right)^{2}}\right),\left(x_{\|}-y_{\|},\left|x_{3}-y_{3}\right|\right)\right)\right\}}{2 i \sqrt{E-\left(k_{\|}+2 \pi n_{\|}\right)^{2}}},
\end{aligned}
$$

где под $\sqrt{z}$ для $z=\rho e^{i \phi}$ понимаем $\sqrt{\rho} e^{i \phi / 2}$.

В настояшей работе строится единая теория возмушений для резонансов, а также для собственных значений, находящихся на непрерывном спектре, оператора $H\left(k_{\|}\right)$. Полученные формулы достаточно просты и удобны для использования, а в случае собственных значений переходят в обычные формулы теории возмушений (в отличие от некоторых примеров в [5]). Отсюда вытекает, что поправка первого порядка малости к невозмущенному собственному значению, лежащему на непрерывном спектре, вещественна. Однако, данное собственное значение при возмушении преврашается в общем положении в резонанс. В статье приведено условие, при вьполнении которого поправка первого порядка малости к соответствуюшей невозмушенной собственной функции остается лежать в $L^{2}(\Omega)$.

Ранее в книге [6] (см. главу 3) была получена формула для возмушенного резонанса в случае центрально-симметричного потенциала. В работе [7] исследовано поведение собственных значений (превращающихся в резонансы) на границе непрерывного спектра для потенциала, экспоненциально убывающего при $|x| \rightarrow \infty$. Аналогичное исследование для периодического потеншиала, возмушенного функцией, периодической по двум переменным и убываюшей по третьей переменной, проведено в статье [8]. Обшие результаты, относяшиеся к возмушениям операторов, проблемам алгебраической и геометрической кратности резонансов и возможности их разложения в ряды Пюизё по малому параметру см. в работах [5], [9]. В статьях [10], [11] (см. также [12], Приложение В) изучаются кратности резонансов, а также их асимптотика в терминах модифицированных определителей Фредгольма операторов, естественно возникаюших при определении резонансов (см. замечание 1).

Через $C, c_{0}$ и т.п. в статье обозначаются константы.

\section{2. ПОНЯТИЕ РЕЗОНАНСА}

Уравнение на собственные значения $H\left(k_{\|}\right) \psi=E \psi$ для оператора $H\left(k_{\|}\right)$заменим для $E<k_{\|}^{2}$ эквивалентным уравнением $\psi=-R_{0}\left(k_{\|}, E\right) V \psi$ или, полагая $\phi=\sqrt{|V|} \psi$, уравнением

$$
\phi(x)=-\int_{\Omega} \sqrt{|V(x)|} G_{0}\left(x-y, k_{\|}, E\right) \sqrt{V(y)} \phi(y) d y,
$$

где $\sqrt{V}=\operatorname{sgn} V \cdot \sqrt{|V|}$.

Предположим, что

$$
k_{\|}^{2}<\operatorname{Re} E<\left(k_{\|}+2 \pi n_{\|}\right)^{2}, \quad n_{\|} \neq 0 .
$$


Положим $k_{3}=\sqrt{E-k_{\|}^{2}}$, тогда

$$
\sqrt{|V(x)|} G_{0}\left(x-y, k_{\|}, E\right) \sqrt{V(y)}=\sqrt{|V(x)|} G_{0}(x-y, k) \sqrt{V(y)}
$$

оказывается мероморфной $L^{2}(\Omega \times \Omega)$-значной функцией параметра $k_{3}$ с полюсом порядка единица в нуле [4]. При этом величинам $k_{3}$ с положительной мнимой частью отвечает $E=k_{\|}^{2}+k_{3}^{2}$ на первом листе римановой поверхности функции $G_{0}$ и, вместе с тем, экспоненциальное убывание $G_{0}$ при $\left|x_{3}-y_{3}\right| \rightarrow \infty$, а величинам $k_{3}$ с отрицательной мнимой частью отвечает $E$ на втором ("нефизическом") листе и экспоненциальное возрастание $G_{0}$ при $\left|x_{3}-y_{3}\right| \rightarrow \infty$ (см. в [4] свойства функции $\left.G_{0}\right)$. Убывание или возрастание $G_{0}$ приводит в силу равенства

$$
\psi(x)=-\int_{\Omega} G_{0}(x-y, k) V(y) \psi(y) d y
$$

к экспоненциальному убыванию или возрастанию при $\left|x_{3}\right| \rightarrow \infty$ (такому же, как у $\left.e^{i\left(k,\left(x_{\|},\left|x_{3}\right|\right)\right)}\right)$ ненулевых решений уравнения (6) (см. [4], [13]). При этом вследствие самосопряженности оператора $H\left(k_{\|}\right)$убываюшим решениям отвечают $E \in \mathbb{R}$. В случае возрастаюших решений $E$ называют резонансом; при этом величина $\operatorname{Im} E \neq 0$, обратная времени жизни резонансного состояния [6], и, следовательно, величина $\operatorname{Im} k_{3}$ должны быть достаточно малыми. Поэтому уравнение (3), рассматриваемое в классе $L^{2}(\Omega)$, в силу оценки (1) позволяет находить как собственные значения, так и резонансы. В случае $\operatorname{Re} E>\left(k_{\|}+2 \pi n_{\|}^{(0)}\right)^{2}$, где значение $n_{\|}^{(0)} \neq 0$ доставляет минимум разности $\operatorname{Re} E-\left(k_{\|}+2 \pi n_{\|}^{(0)}\right)^{2}$ среди всех таких неотрицательных разностей, полагаем $k_{3}=\sqrt{E-\left(k_{\|}+2 \pi n_{\|}^{(0)}\right)^{2}}$ и приходим к тем же утверждениям, что и выше. В этом случае выкладки несколько более громоздкие, поэтому в дальнейшем для простоты предполагаем, что выполнено условие (4).

С учетом вышесказанного и специфики функции $G_{0}$ в “пленочном" случае следуюшее определение резонанса согласуется с обычными определениями, приведенными в книгах $[6],[12]$.

ОПРЕДЕЛЕНИЕ 1 . Назовем резонансом такое значение $k_{3}$ (или соответствующее значение $E$ ), для которого имеет место неравенство $\operatorname{Im} k_{3}<0$ и сушествует ненулевое решение $\phi \in L^{2}(\Omega)$ уравнения (3).

ОПРЕДЕЛЕНИЕ 2. Назовем уровнем собственное значение или резонанс.

Уравнение, сопряженное к (3), имеет вид

$$
\phi^{*}(x)=-\int_{\Omega} \sqrt{V(x)} G_{0}^{-}\left(x-y, k^{*}\right) \sqrt{|V(y)|} \phi^{*}(y) d y
$$

где $k^{*}=\left(k_{\|}, \overline{k_{3}}\right)$, и в случае $n_{\|}=0 G_{0}^{-}$по сравнению с $G_{0}$ из формулы $(2)$ имеет корни противоположного знака. Вследствие теоремы Фредгольма пространства решений 
уравнений $(3)$ и (7) имеют одну и ту же размерность $d<\infty-$ (геометрическую) кратность уровня.

В дальнейшем через $\sqrt{|V|} R_{0}(k) \sqrt{V}$ будем обозначать не только “окаймленную" резольвенту оператора $H_{0}\left(k_{\|}\right)$, но и, в более общем смысле, компактный оператор с ядром (5), который определен как оператор в $L^{2}(\Omega)$, в частности, в точках $k_{3}$, соответствуюших значениям $E$ из окрестности множества $\left[k_{\|}^{2}, \infty\right) \backslash\left\{\left(k_{\|}+2 \pi n_{\|}\right)^{2}, n_{\|} \in \mathbb{Z}^{2}\right\}[4]$.

Вследствие резольвентного тождества

$$
1-\sqrt{|V|} R(k) \sqrt{V}=\left(1+\sqrt{|V|} R_{0}(k) \sqrt{V}\right)^{-1}
$$

и аналитической теоремы Фредгольма [14] операторнозначная функция $\sqrt{|V|} R(k) \sqrt{V}$ мероморфно продолжается в окрестности точек $k_{3}$, для которых соответствующие значения $E$ принадлежат существенному спектру $\left[k_{\|}^{2}, \infty\right)$ оператора $H\left(k_{\|}\right)$; значения данной функции остаются компактными операторами, которые будем обозначать тем же символом. При этом всякий уровень $k_{3}$ является полюсом мероморфной по $k_{3}$ операторнозначной функции $\sqrt{|V|} R(k) \sqrt{V}$, причем коэффициенты при отрицательных степенях ее разложения в ряд Лорана - это операторы конечного ранга [14].

Всюду в дальнейшем, за исключением раздела 4 и теоремы 7 , предполагаем, что полюс функции $\sqrt{|V|} R(k) \sqrt{V}$ в точке резонанса $k_{3}=k_{3}^{(0)}$ имеет порядок единица (достаточные условия этого см. в замечании 1 и в теоремах 3,7$)$, так что

$$
\sqrt{|V|} R(k) \sqrt{V}=\frac{1}{k_{3}-k_{3}^{(0)}} \sum_{n=1}^{n_{0}} c_{n}\left(\cdot, \phi_{n}^{*}\right) \phi_{n}+Q(k) ;
$$

здесь $c_{n}, n=1, \ldots, n_{0}$ - некоторые константы; $Q(k)$ - аналитическая по $k_{3}$ в окрестности точки $k_{3}^{(0)}$ операторнозначная функция; как функции $\left\{\phi_{n}^{*}\right\}_{n=1}^{n_{0}}$, так и функции $\left\{\phi_{n}\right\}_{n=1}^{n_{0}}$ будем без ограничения общности считать линейно независимыми. Положим $k_{0}=\left(k_{\|}, k_{3}^{(0)}\right)$.

ЛЕмма 1. Функиии $\phi_{n}$ являются решениями уравнения (3) в точке $k_{0}$, а функчии $\phi_{n}^{*}$ - решениями сопряжсенного уравнения (7) в точке $k_{0}^{*}$.

ДокАЗАТЕльСтво. В силу (8), (9)

$$
\left(1+\sqrt{|V|} R_{0}(k) \sqrt{V}\right)^{-1}=1-\frac{1}{k_{3}-k_{3}^{(0)}} \sum_{n=1}^{n_{0}} c_{n}\left(\cdot, \phi_{n}^{*}\right) \phi_{n}-Q(k) .
$$

Вследствие (4) резольвента $R_{0}(k)$ аналитична в окрестности точки $k_{3}^{(0)}$. Умножая обе части равенства $(10)$ слева на $\left(k_{3}-k_{3}^{(0)}\right)\left(1+\sqrt{|V|} R_{0}(k) \sqrt{V}\right)$ и переходя к пределу при $k_{3} \rightarrow k_{3}^{(0)}$, получаем соотношение

$$
\sum_{n=1}^{n_{0}} c_{n}\left(\cdot, \phi_{n}^{*}\right)\left(1+\sqrt{|V|} R_{0}(k) \sqrt{V}\right) \phi_{n}=0,
$$

откуда следует требуемьй результат для функций $\phi_{n}$. Для доказательства аналогичного утверждения для функций $\phi_{n}^{*}$ нужно обе части равенства $(10)$ умножить на $\left(k_{3}-\right.$ $\left.k_{3}^{(0)}\right)\left(1+\sqrt{|V|} R_{0}(k) \sqrt{V}\right)$ справа. Лемма доказана. 
СлеДСтвиЕ 1. Справедливо неравенство $n_{0} \leqslant d$.

СлЕдСтвиЕ 2. Функиии $\psi_{n}, \psi_{n}^{*}$, определяемье равенствами $\sqrt{|V|} \psi_{n}=\phi_{n}$, $\sqrt{V} \psi_{n}^{*}=\phi_{n}^{*}$ и уравнениями (3), (7), удовлетворяют уравнениям Шредингера $H\left(k_{\|}\right) \psi_{n}=E_{0} \psi_{n}, H\left(k_{\|}\right) \psi_{n}^{*}=E_{0}^{*} \psi_{n}^{*}$, әде $E=k_{0}^{2}, E^{*}=k_{0}^{* 2}$.

ЗАМЕчАниЕ 1. Уровни определяются уравнением вида $\Delta(k)=0$, где $\Delta(k)$ не равна тождественно нулю по $k_{3}$, является аналитической функцией аргумента $k$ [4], [14] и с точностью до умножения на ненулевую аналитическую функцию совпадает с (регуляризованным) определителем Фредгольма $\operatorname{det}_{2}\left(1+\sqrt{|V|} R_{0}(k) \sqrt{V}\right)$ (см. [15]). Согласно определению из книги [12] алгебраическая кратность $\kappa$ резонанса (уровня) есть порядок нуля по $k_{3}$ определителя Фредгольма или, что то же самое, порядок нуля функции $\Delta(k)$ в точке $k_{3}^{(0)}$. Вследствие подготовительной теоремы Вейерштрасса [16] в окрестности $k_{3}^{(0)}$ имеется $\kappa$, возможно, сливаюшихся уровней. Наконец, из доказательства аналитической теоремы Фредгольма [14] вытекает, что порядок полюса резольвенты $R(k)$ в точке $k_{3}^{(0)}$ не превосходит $\kappa$ (см. также [9]).

Заметим, что у уровней нет конечных точек сгущения. Это вытекает из мероморфной теоремы Фредгольма [1] и результата работы [4] о малости функции $\sqrt{|V|} G_{0} \sqrt{V}$ при некоторых $k$.

\section{3. ВОЗМУЩЕНИЕ УРОВНЕЙ КРАТНОСТИ ЕДИНИЦА}

В этом разделе полагаем $d=1$. Тогда (см. предыдуший раздел)

$$
\sqrt{|V|} R(k) \sqrt{V}=\frac{c_{0}\left(\cdot, \phi_{0}^{*}\right) \phi_{0}}{k_{3}-k_{3}^{(0)}}+Q(k) .
$$

Заметим, что из этого равенства для достаточно малых $\epsilon>0$ вытекает формула

$$
c_{0}=\frac{1}{2 \pi i\left\|\phi_{0}^{*}\right\|^{2}\left\|\phi_{0}\right\|^{2}} \int_{\left|k_{3}-k_{3}^{(0)}\right|=\epsilon}\left(\phi_{0}, \sqrt{|V|} R(k) \sqrt{V} \phi_{0}^{*}\right) d k_{3} .
$$

По поводу вычисления $c_{0}$ в частном случае см. теорему 3 ниже.

Введем обозначения:

$$
H_{1}=\left\{\sqrt{V} \psi: \psi \in L^{2}(\Omega)\right\}, \quad H_{-1}=\left\{\psi: \sqrt{V} \psi \in L^{2}(\Omega)\right\} .
$$

Всюду в дальнейшем предполагаем, что $W(x)$ - вешественная функция такая, что $W(x) / V(x) \in L^{\infty}(\Omega)$. Положим $H_{\epsilon}\left(k_{\|}\right)=H\left(k_{\|}\right)+\epsilon W(x)$.

Лемма 2. Пусть $\epsilon \neq 0$ вещественно. Тогда если

$$
k_{3}^{2} \neq\left(k_{\|}+2 \pi n_{\|}\right)^{2}-k_{\|}^{2}, \quad n_{\|} \in \mathbb{Z}^{2},
$$

то всякое решение из $\mathrm{H}_{-1}$ уравнения

$$
\psi=-\epsilon R(k) W \psi
$$

является решением уравнения

$$
\psi=-R_{0}(k)(V+\epsilon W) \psi .
$$

Если $k_{3} \approx$ тому же не является уровнем оператора $H\left(k_{\|}\right)$, то уравнения (13) и (14) әквивалентны в классе функций $H_{-1}$. 
ДоКАЗАТЕльство. Операторы $\sqrt{|V|} R_{0}(k) \sqrt{V}, \sqrt{|V|} R(k) \sqrt{V}$ действуют в $L^{2}(\Omega)$, поэтому $R_{0}(k), R(k)$ можно рассматривать как операторы, действуюшие из $H_{1}$ в $H_{-1}$. Следовательно, преобразуемый ниже оператор действует в $H_{-1}$ :

$$
\begin{aligned}
1+R_{0}(k)(V+\epsilon W) & =\left(1+R_{0}(k) V\right)\left(1+\epsilon\left(1+R_{0}(k) V\right)^{-1} R_{0}(k) W\right)= \\
& =\left(1+R_{0}(k) V\right)(1+\epsilon R(k) W) .
\end{aligned}
$$

Из полученного равенства вытекает доказываемое утверждение.

Везде в дальнейшем предполагаем, что $k_{3}^{2} \neq\left(k_{\|}+2 \pi n_{\|}\right)^{2}-k_{\|}^{2}, n_{\|} \in \mathbb{Z}^{2}$, а там, где говорится о возмушенном функшией $\epsilon W$ уровне, в качестве уравнения, определяюшего уровни, используем уравнение (13).

Делая в (13) замену $\phi_{1}=\sqrt{|W|} \psi$, получаем уравнение

$$
\phi_{1}=-\epsilon \sqrt{|W|} R(k) \sqrt{W} \phi_{1},
$$

которое будем рассматривать в классе $L^{2}(\Omega)$. Функции $\psi$ такие, что $\sqrt{W} \psi \in L^{2}(\Omega)$, образуют, вообше говоря, более широкий класс, чем $H_{-1}$, но это не приводит к расширению понятия уровня, поскольку в силу (13) функция $\psi$, отвечаюшая решению $\phi_{1}$, принадлежит классу $H_{-1}$ и согласно лемме 2 определяет уровень.

В следуюшей теореме функции $\psi_{0}, \psi_{0}^{*}$ определяются, как в следствии 2 для $n_{0}=1$.

ТЕорема 1. Предположим, что $k_{3}^{(0)}$ является уровнем геометрической кратности единица оператора $H\left(k_{\|}\right)$. Тогда для всех достаточно малых $\epsilon$ существует уровень $k_{3}^{(\epsilon)}=k_{3}^{(\epsilon)}\left(k_{\|}\right)$оператора $H_{\epsilon}\left(k_{\|}\right)$геометрической кратности единица, аналитически зависящий от $\epsilon$, причем

$$
k_{3}^{(\epsilon)}=k_{3}^{(0)}-\epsilon c_{0}\left(W \psi_{0}, \psi_{0}^{*}\right)+o(\epsilon)
$$

и, соответственно, для $E_{\epsilon}=k_{\|}^{2}+\left(k_{3}^{(\epsilon)}\right)^{2}$

$$
E_{\epsilon}=E_{0}-2 \epsilon c_{0} k_{3}^{(0)}\left(W \psi_{0}, \psi_{0}^{*}\right)+o(\epsilon)
$$

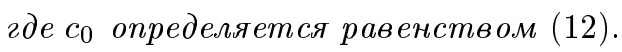

ДоКАЗАТЕЛЬСТво. Из формул (15), (11) получаем, полагая $v=\sqrt{W / V}$, уравнение, определяюшее уровни для $\epsilon \neq 0$,

$$
\phi_{1}=-\epsilon|v| \sqrt{|V|} R(k) \sqrt{V} v \phi_{1}=-\frac{\epsilon c_{0}\left(v \phi_{1}, \phi_{0}^{*}\right)|v| \phi_{0}}{k_{3}-k_{3}^{(0)}}-\epsilon|v| Q(k) v \phi_{1} .
$$

Отсюда находим алгебраическое уравнение, определяюшее уровни,

$$
k_{3}=k_{3}^{(0)}-\epsilon c_{0}\left((1+\epsilon|v| Q(k) v)^{-1}\left(|v| \phi_{0}\right), v \phi_{0}^{*}\right) .
$$


Операторнозначную функцию

$$
(1+\epsilon|v| Q(k) v)^{-1}
$$

можно разложить в равномерно сходяшийся ряд по степеням $\epsilon$. Вследствие теоремы Вейерштрасса об аналитичности равномерно сходяшегося ряда из аналитических функций правая часть равенства (19) представляет собой аналитическую функцию по совокупности переменных $\left(k_{3}, \epsilon\right)$. Из теоремы о неявной функции [16] и соотношения (19) для достаточно малых $\epsilon$ вытекает сушествование решения данного уравнения $k_{3}=k_{3}^{(\epsilon)}$, аналитически зависящего от $\epsilon$. Формула (16) следует из (19) и упомянутого разложения выражения функции (20) в ряд. Кратность уровня $k_{3}^{(\epsilon)} \neq k_{3}^{(0)}$ равна единице в силу (18). Теорема доказана.

ТЕОРема 2. Предположим, что геометрическая кратность уровня $k_{3}^{(0)}$ равна единице. Тогда общее решение $\psi$ в классе $H_{-1}$ уравнения (15) для всех достаточно мальх $|\epsilon|$ имеет вид

$$
\psi=C\left(\psi_{0}-\epsilon Q_{0}(k)\left(W \psi_{0}\right)\right)+o(\epsilon)
$$

где $Q_{0}(k)=|V|^{-1 / 2} Q(k) V^{-1 / 2}$, а $Q(k)$ определяется равенством (11) (из этого равенства видно, что умножение на $|V|^{-1 / 2}$ u $V^{-1 / 2}$ имеет смысл); о $\epsilon$ ) понимается в смислле $\|\sqrt{W} o(\epsilon)\| \epsilon^{-1} \rightarrow 0$ при $\epsilon \rightarrow 0$.

ДокАЗАТЕЛьство. Вследствие уравнения (18) имеем

$$
\phi_{1}=C(1-\epsilon|v| Q(k) v)\left(|v| \phi_{0}\right)+o(\epsilon),
$$

откуда после перехода к функциям $\psi$ и $\psi_{0}$ и вытекает утверждение теоремы.

\section{4. ВОЗМУЩЕНИЕ СОБСТВЕННОГО ЗНАЧЕНИЯ КРАТНОСТИ ЕДИНИЦА НА НЕПРЕРЫВНОМ СПЕКТРЕ}

Лемма 3. Пусть значение Е вещественно и не совпадает $c\left(k_{\|}+2 \pi n_{\|}\right)^{2}, n_{\|} \in$ $\mathbb{Z}^{2}$. Блоховская по переменным $x_{1}, x_{2}$ функиия $\psi$ такая, что $\sqrt{V} \psi \in L^{2}(\Omega)$, удовлетворяет уравнению Шредингера $(-\Delta+V) \psi=E \psi$ тогда и только тогда, когда $\psi$ удовлетворяет уравнению

$$
\psi(x)=\psi_{0}(x)-\int_{\Omega} G_{0}\left(x-y, k_{\|}, E\right) V(y) d y
$$

$2 \partial e$

$$
\psi_{0}(x)=\sum_{\substack{E-\left(k_{\|}+2 \pi n_{\|}\right)^{2}>0 \\ j=1,2}} c_{n_{\|}}^{(j)} \exp \left\{i\left(\left(k_{\|}+2 \pi n_{\|},(-1)^{j} \sqrt{E-\left(k_{\|}+2 \pi n_{\|}\right)^{2}}\right), x\right)\right\} .
$$

При этом

$$
\psi(x)=\psi_{0}(x)+\sum_{E-\left(k_{\|}+2 \pi n_{\|}\right)^{2}>0} a_{n_{\|}}^{(l)} \exp \left\{i\left(\left(k_{\|}+2 \pi n_{\|},(-1)^{l} \sqrt{E-\left(k_{\|}+2 \pi n_{\|}\right)^{2}}\right), x\right)\right\}+\eta(x),
$$


$2 \partial e$

$$
\begin{aligned}
a_{n_{\|}}^{(l)}= & \frac{1}{2 i \sqrt{E-\left(k_{\|}+2 \pi n_{\|}\right)^{2}}} \times \\
& \times \int_{\Omega} \exp \left\{i\left(\left(k_{\|}+2 \pi n_{\|},(-1)^{l+1} \sqrt{E-\left(k_{\|}+2 \pi n_{\|}\right)^{2}}\right), x\right)\right\} V(x) \psi(x) d x ;
\end{aligned}
$$

здесь $l=0$ для $x_{3}>0$ и $l=1$ для $x_{3}<0$, а функция $\eta(x)$ удовлетворяет оченке

$$
|\eta(x)| \leqslant C e^{-b\left|x_{3}\right|}, \quad b>0
$$

ДокАЗАТЕльство. Доказательство первого утверждения леммы проводится стандартно, доказательство второго утверждения содержится в доказательстве теоремы 2 в [4].

СЛЕДСТВИЕ 3. В условиях леммы 3 включение $\psi \in L^{2}(\Omega)$, во-первьх, эквивалентно тому, что $\psi_{0}=0$, и во-вторьх, әквивалентно тому, что $a_{n_{\|}}^{(l)}=0$ для $l=0,1$ и всех $n_{\|}$maких, что $E-\left(k_{\|}+2 \pi n_{\|}\right)^{2}>0$.

Лемма 4. Пусть в условиях леммы $3 E>k_{\|}^{2} u c_{n_{\|}}^{(1)}=0$ для всех $n_{\|}$таких, что $E-\left(k_{\|}+2 \pi n_{\|}\right)^{2}>0$. Тогда

$$
\sum_{E-\left(k_{\|}+2 \pi n_{\|}\right)^{2}>0} \sqrt{E-\left(k_{\|}+2 \pi n_{\|}\right)^{2}}\left(\left|a_{n_{\|}}^{(1)}\right|^{2}+\left|c_{n_{\|}}^{(0)}+a_{n_{\|}}^{(0)}\right|^{2}-\left|c_{n_{\|}}^{(0)}\right|^{2}\right)=0 .
$$

ДокАЗАТЕЛЬСтво. Из леммы 3 и результатов работы [17] (см. теорему 2.7) вытекает, что

$$
\left\|\frac{\partial \eta}{\partial x_{3}}\left(x+\left(0,0, n_{3}\right)\right)\right\|_{L^{2}(0,1)^{3}} \rightarrow 0, \quad\left|n_{3}\right| \rightarrow \infty .
$$

Требуемое утверждение теперь легко получить, применяя формулы Грина.

СледСТВИЕ 4. Всякий вещественный уровень является собственным значениeм.

ЛЕмма 5. Пусть $E>k_{\|}^{2}, \psi$-решение уравнения (22) при $\psi_{0}=0$, принадлежащее тому же классу, что и в лемме 3 . Тогда имеет место оиенка

$$
\mid \psi(x) \leqslant C e^{-\delta\left|x_{3}\right|}
$$

где $\delta=\min \{a, \sigma\}$, значение а взято из неравенства (1), а $\sigma>0$ зависит лишь от $k_{\|} u E$. 
ДокАЗАтЕльСтво. Имеем включение $\psi_{0} \in L^{\infty}(\Omega)$ (доказательство см. в [4]). Согласно лемме $4 a_{n_{\|}}^{(j)}=0$ для $j=0,1$ и всех $n_{\|}$таких, что $E-\left(k_{\|}+2 \pi n_{\|}\right)^{2}>0$. Далее оцениваем модуль $\psi(x)$ через правую часть уравнения (22), пользуясь равенством Парсеваля при оценке интеграла по $y_{\|} ;$начиная с достаточно больших $\left|n_{\|}\right|$сумму по $n_{\|} \in \mathbb{Z}^{2}$ следует заменить интегралом. Полученные в результате интегралы оцениваются элементарно (см. подобные выкладки в [4]).

ТЕОрема 3. Пусть $E_{0}>k_{\|}^{2}$ - собственное значение кратности единица. Предположим, что выполняются неравенства

$$
C_{1} e^{-b\left|x_{3}\right|} \leqslant|V(x)| \leqslant C_{2} e^{-a\left|x_{3}\right|},
$$

где $0<b / 2<a \leqslant b<2 \sigma$ ( $\sigma$ взято из леммы 5). Тогда операторнозначная функция $\sqrt{|V|} R(k) \sqrt{V}$ в окрестности точки $k_{3}^{(0)}=\sqrt{E_{0}-k_{\|}^{2}}$ имеет вид

$$
\sqrt{|V|} R(k) \sqrt{V}=-\frac{\left(\cdot, \phi_{0}^{*}\right) \phi_{0}}{2 k_{3}^{(0)}\left(k_{3}-k_{3}^{(0)}\right)}+Q(k),
$$

где $Q(k)$ аналитически зависит от $k_{3}, \phi_{0}=\sqrt{|V|} \psi_{0}, \phi_{0}^{*}=\sqrt{V} \psi_{0}$, а $\psi_{0} \in L^{2}(\Omega)$ нормированная собственная функиия оператора $H\left(k_{\|}\right)$, отвечающая собственному значению $E_{0}$.

ДоказательСтво. Обозначим через $P_{\mathrm{p}}$ и $P_{\mathrm{c}}$ ортопроекторы на подпространства $\mathcal{H}_{\mathrm{p}}$ и $\mathcal{H}_{\mathrm{c}}$, соответственно, отвечаюшие точечному и непрерывному спектру оператора $H\left(k_{\|}\right)[18]$. Для $E$ из резольвентного множества оператора $H\left(k_{\|}\right)$имеем

$$
\begin{aligned}
R\left(k_{\|}, E\right) & =R(k)=R(k) P_{\mathrm{p}}+R(k) P_{\mathrm{c}}= \\
& =\frac{\left(\cdot, \psi_{0}\right) \psi_{0}}{E_{0}-E}+Q_{\mathrm{p}}\left(k_{\|}, E\right)+R\left(k_{\|}, E\right) P_{\mathrm{c}}
\end{aligned}
$$

где $Q_{\mathrm{p}}\left(k_{\|}, E\right)$ - ортопроектор на собственные векторы, отличные от $\psi_{0}$. Поскольку уровни не имеют точек сгушения (см. раздел 2$), Q_{\mathrm{p}}$ аналитически зависит от $E$ в окрестности $E_{0}$. Далее, из (23) получаем

$$
\begin{aligned}
\sqrt{|V|} R(k) \sqrt{V}= & -\frac{\left(\cdot, \phi_{0}^{*}\right) \phi_{0}}{2 k_{3}^{(0)}\left(k_{3}-k_{3}^{(0)}\right)}+\frac{\left(\cdot, \phi_{0}^{*}\right) \phi_{0}}{2 k_{3}^{(0)}\left(k_{3}+k_{3}^{(0)}\right)}+ \\
& +\sqrt{|V|} Q_{\mathrm{p}}(k) \sqrt{V}+\sqrt{|V|} R(k) P_{\mathrm{c}} \sqrt{V}
\end{aligned}
$$

где $\phi_{0}=\sqrt{|V|} \psi_{0}, \phi_{0}^{*}=\sqrt{V} \psi_{0}$, а все слагаемые в правой части (24) суть мероморфные функции с вычетами, являющимися операторами конечного ранга. Достаточно доказать, что операторнозначная функция $\sqrt{|V|} R(k) P_{\mathrm{c}} \sqrt{V}$ не может иметь полюса в точке $k_{3}^{(0)}$. В предположении противного

$$
\sqrt{|V|} R(k) P_{\mathrm{c}} \sqrt{V}=\sum_{m=1}^{m_{0}} \frac{P_{m}}{\left(k_{3}-k_{3}^{(0)}\right)^{m}}+Q_{\mathrm{c}}(k),
$$


где $P_{m}, m=1, \ldots, m_{0}$, суть операторы конечного ранга, а $Q_{\mathrm{c}}(k)$ аналитична в окрестности $k_{3}^{(0)}$. В силу известной оценки для резольвенты самосопряженного оператора для любых $\psi_{1}, \psi_{2} \in L^{2}(\Omega)$ справедливы неравенства

$$
\left(\sqrt{|V|} R(k) \sqrt{V} \psi_{1}, \psi_{2}\right) \leqslant \frac{C}{|\operatorname{Im} E|} \leqslant \frac{C_{1}}{\left|\operatorname{Im} k_{3}\right|} .
$$

Отсюда и из (25) получаем, что $m_{0}=1$. Далее, из $(24),(25)$ и леммы 1 вытекает, что

$$
\sqrt{|V|} R(k) P_{\mathrm{c}} \sqrt{V}=C_{0} \frac{\left(\cdot, \phi_{0}^{*}\right) \phi_{0}}{k_{3}-k_{3}^{(0)}}+Q_{\mathrm{c}}(k),
$$

откуда

$$
R(k) P_{\mathrm{c}}=C_{0} \frac{\left(\cdot, \psi_{0}\right) \psi_{0}}{k_{3}-k_{3}^{(0)}}+\frac{1}{\sqrt{|V|}} Q_{\mathrm{c}}(k) \frac{1}{\sqrt{V}} .
$$

В силу леммы 5 и условия теоремы

$$
\frac{\left|\psi_{0}(x)\right|}{\sqrt{|V(x)|}} \leqslant C e^{(b / 2-\delta)\left|x_{3}\right|},
$$

причем $b / 2-\delta<0$. Следовательно, $\psi_{0} \in H_{1}$. Положив $E=E^{\prime}+i \epsilon$, где $E^{\prime}>k_{\|}^{2}, \epsilon>0$, и переходя к пределу в (26) на элементе $\psi_{0}$ при $\epsilon \rightarrow 0$ (операторы в (26) можно рассматривать, в том числе и для $\epsilon=0, E^{\prime} \neq E_{0}$, как операторы из $H_{1}$ в $H_{-1}$, см. выше), получим равенство $\left(R(k) P_{\mathrm{c}}\right) \psi_{0}=0$ для $k_{3}>0$. Отсюда вследствие $(26)$ и аналитичности $Q_{\mathrm{c}}(k)$ имеем равенство $C_{0}=0$, что и доказывает теорему.

СЛЕДСТВИЕ 5 . В условиях теоремы $3 c_{0}=-\left(2 k_{3}^{(0)}\right)^{-1}$, так что формула (17) превращается в объчную формулу теории возмущений.

СлеДСТВИЕ 6. В силу (17) имеем $\operatorname{Im} E_{\epsilon}=o\left(\epsilon^{2}\right)$. Таким образом, время жсизни резонансного состояния [6] есть $O\left(\epsilon^{-2}\right)$.

ТЕОРема 4. Пусть $\psi_{0}=\psi_{0}\left(x, k_{\|}\right) \in L^{2}(\Omega)$ - собственная функиия оператора $H\left(k_{\|}\right)$, отвечающая собственному значению $E_{0}>k_{\|}^{2}$ кратности единица. Тогда первая по порядку малости є поправка

$$
\psi_{1}=-\epsilon Q_{0}(k)\left(W \psi_{0}\right)
$$

$\kappa \psi_{0}\left(\right.$ см. теорему 2) принадлежит $L^{2}(\Omega)$ в том и только в том случае, когда

$$
\int_{\Omega} W(x) \psi_{0}\left(x, k_{\|}\right) \overline{\psi^{ \pm}(x, k)} d x=0
$$

где $\psi^{ \pm}(x, k)$ удовлетворяют уравнениям типа Липпмана-Швингера

$$
\psi^{ \pm}(x, k)=e^{i\left(k^{ \pm}, x\right)}-\int_{\Omega} G_{0}\left(x-y, k^{-}\right) V(y) \psi^{ \pm}(y, k) d y, \quad k^{ \pm}=\left(k_{\|}, \pm k_{3}\right)
$$


ДокАЗАТЕЛЬСТво. Пользуясь формулой (11), условие

$$
\psi_{1}=-\epsilon \frac{1}{\sqrt{|V|}} Q(k) \frac{1}{\sqrt{V}}\left(W \psi_{0}\right)=-\left(R(k)-c_{0} \frac{\left(\cdot, \psi_{0}\right) \psi_{0}}{k_{3}-k_{3}^{(0)}}\right)\left(W \psi_{0}\right) \in L^{2}(\Omega)
$$

перепишем в виде

$$
R(k)\left(W \psi_{0}\right) \in L^{2}(\Omega)
$$

Имеем

$$
\begin{aligned}
R(k) W \psi_{0} & =\left(R_{0}(k)-R_{0}(k) V R(k)\right)\left(W \psi_{0}\right)= \\
& =R_{0}(k)(1-V R(k))\left(W \psi_{0}\right) .
\end{aligned}
$$

Следовательно (см. доказательство леммы 3), условие (27) имеет место тогда и только тогда, когда справедливо равенство

$$
\int_{\Omega} e^{-i\left(k^{ \pm}, x\right)}(1-V R(k))\left(W \psi_{0}\right) d x=0 .
$$

Вьполним преобразования

$$
\begin{aligned}
(1-V R(k))\left(W \psi_{0}\right) & =\sqrt{V}(1-\sqrt{|V|} R(k) \sqrt{V})\left(W \psi_{0} / \sqrt{V}\right)= \\
& =\sqrt{V}\left(1+\sqrt{|V|} R_{0}(k) \sqrt{V}\right)^{-1}\left(W \psi_{0} / \sqrt{V}\right) .
\end{aligned}
$$

Таким образом, равенство (28) можно переписать в виде

$$
\begin{aligned}
((1 & \left.\left.+\sqrt{|V|} R_{0}(k) \sqrt{V}\right)^{-1}\left(W \psi_{0} / \sqrt{V}\right), \sqrt{V} e^{i\left(k^{ \pm}, x\right)}\right)= \\
& =\left(W \psi_{0} / \sqrt{V},\left(1+\sqrt{V} R_{0}\left(k^{-}\right) \sqrt{|V|}\right)^{-1}\left(\sqrt{V} e^{i\left(k^{ \pm}, x\right)}\right)\right)= \\
& =\left(\psi_{0}, W \psi^{ \pm}(x, k)\right)=0 .
\end{aligned}
$$

Теорема доказана.

ЗАмЕчанИЕ 2. Из теоремы 4 вытекает, что собственное значение на непрерывном спектре при возмущении превращается, как правило, в резонанс, поскольку возмущенная собственная функция в общем положении не принадлежит $L^{2}(\Omega)$.

\section{5. УРОВНИ КРАТНОСТИ, БОЛЬШЕЙ ЕДИНИЦЫ}

В данном разделе предполагаем, что выполнено (9).

Выберем функции $\psi_{n}, \psi_{n}^{*} \in L^{2}(\Omega), \quad n=1, \ldots, n_{0}$, которые обладают свойством $\left(\psi_{n}, \phi_{m}\right)=\delta_{n m},\left(\psi_{n}^{*}, \phi_{m}^{*}\right)=\delta_{n m}$, где $\delta_{n m}-$ символ Кронекера, $n, m=1, \ldots, n_{0}$. Тогда из (9) получаем формулы для $c_{n}$ :

$$
c_{n}=\frac{1}{2 \pi i} \int_{\left|k_{3}-k_{3}^{(0)}\right|=\epsilon}\left(\psi_{n}, \sqrt{|V|} R(k) \sqrt{V} \psi_{n}^{*}\right) d k_{3},
$$

где $\epsilon>0$ достаточно мало.

В следующей теореме предполагаем, что функции $\sqrt{W / V} \phi=v \phi_{n}, n=1, \ldots, n_{0}$, сохраняют линейную независимость. Алгебраическая кратность уровня понимается как порядок нуля $\operatorname{det}_{2}(1+\sqrt{|W|} R(k) \sqrt{W})$. 
Tеорема 5. Oператор $H_{\epsilon}\left(k_{\|}\right)$имеет в окрестности точки $k_{3}^{(0)}$ ровно $n_{0}$ уровней (возможно, сливающихся для конечного набора значений $\epsilon$ ), которые разлагаются в ряды Пюизё по $\epsilon$. В точке $k_{3}^{(0)}$ алгебрачческая и геометрическая кратности уровня совпадают и равны $n_{0}$.

ДокАЗАТЕЛЬСтво. Как и при доказательстве теоремы 1 , имеем уравнение в $L^{2}(\Omega)$ для определения уровней:

$$
\phi_{1}=-\frac{\epsilon}{k_{3}-k_{3}^{(0)}} \sum_{n=1}^{n_{0}} c_{n}\left(v \phi_{1}, \phi_{n}^{*}\right)|v| \phi_{n}-\epsilon|v| Q(k) v \phi_{1} .
$$

Отсюда получаем линейную систему

$$
C_{n}=-\frac{\epsilon c_{n}}{k_{3}-k_{3}^{(0)}} \sum_{n^{\prime}=1}^{n_{0}} C_{n^{\prime}}\left((1+\epsilon|v| Q(k) v)^{-1}\left(|v| \phi_{n^{\prime}}\right), v \phi_{n}^{*}\right), \quad n=1, \ldots, n_{0},
$$

а также условие сушествования уровня

$$
\operatorname{det}\left[\delta_{n n^{\prime}}\left(k_{3}-k_{3}^{(0)}\right)+\epsilon c_{n}\left((1+\epsilon|v| Q(k) v)^{-1}\left(|v| \phi_{n^{\prime}}\right), v \phi_{n}^{*}\right)\right]_{n, n^{\prime}=1}^{n_{0}}=0 .
$$

Последнее равенство запишем в виде

$$
\left(k_{3}-k_{3}^{(0)}\right)^{n_{0}}+\epsilon\left(k_{3}-k_{3}^{(0)}\right)^{n_{0}-1} \sum_{n=1}^{n_{0}} c_{n}\left(\psi_{n^{\prime}}, W \psi_{n}^{*}\right)+o(\epsilon)=0 .
$$

Из подготовительной теоремы Вейерштрасса [16] вытекает, что сушествует $n_{0}$ решений этого уравнения, а значит, $n_{0}$ уровней в окрестности точки $k_{3}^{(0)}$. При этом уровни могут сливаться лишь в случае обрашения в нуль результанта, являюшегося ненулевой аналитической функцией аргумента $\epsilon$, откуда следует конечность числа точек слияния в окрестности $\epsilon=0$. Разложимость решений уравнения (32) в ряды Пюизё вытекает из подготовительной теоремы Вейерштрасса и из утверждений, приведенных в книге [1], раздел 12.

Наконец, согласно рассуждению, приведенному в работе [15], определитель в левой части уравнения (31) с точностью до множителя, являющегося ненулевой аналитической функцией от $k_{3}$, совпадает с $\operatorname{det}_{2}(1+\sqrt{|V|} R(k) \sqrt{V})$ и, кроме того, совпадает с определителем матрицы в доказательстве аналитической теоремы Фредгольма [14], поэтому алгебраическая кратность уровня равна $n_{0}$. С другой стороны, геометрическая кратность $d$ уровня есть $n_{0}$ минус ранг упомянутой матрицы [14], т.е. не превосходит $n_{0}$. Но в силу следствия $1 n_{0} \leqslant d$. Теорема полностью доказана.

СлЕДСТВИЕ 7. Пусть $n_{0}=2$, тогда имеет место следующая формула для уровней:

$$
k_{3}^{(1,2)}=k_{3}^{(0)}+\left(\frac{\epsilon}{2}\right)\left(-c_{1} a_{11}-c_{2} a_{22} \pm \sqrt{\left(c_{1} a_{11}-c_{2} a_{22}\right)^{2}+4 c_{1} c_{2}\left|a_{12}\right|^{2}}\right)+o(\epsilon),
$$

где $a_{i j}=\left(\psi_{i}, W \psi_{j}\right), \quad|o(\epsilon)| \leqslant C \epsilon^{2}$ равномерно по $k_{3}$ из окрестности $k_{3}^{(0)}$. 
ТЕОРема 6. В условиях теоремы 5 общее решение $\psi$ уравнения (15) для всех достаточно малых $|\epsilon|$ имеет вид

$$
\psi=\sum_{n=1}^{n_{0}} C_{n} \psi_{n}-\epsilon Q_{0}(k) \sum_{n=1}^{n_{0}} C_{n} W \psi_{n}+o(\epsilon)
$$

где $C_{n}$ удовлетворяют системе $(30)$, а о $(\epsilon)$ понимается так же, как и в теореме 2 .

ДоКАЗАТЕЛЬСТво следует из формул (18) и (29) (см. доказательство теоремы 2).

ТЕОрема 7. Предположим, что собственное значение $E_{0}>k_{\|}^{2}$ имеет кратность d. Пусть выполнены условия

$$
C_{1} e^{-b\left|x_{3}\right|} \leqslant|V(x)| \leqslant C_{2} e^{-a\left|x_{3}\right|},
$$

где $0<b / 2<a \leqslant b<2 \sigma$ ( $\sigma$ взято из леммы 5$)$. Тогда операторнозначная функиия $\sqrt{|V|} R(k) \sqrt{V}$ в окрестности точки $k_{3}^{(0)}=\sqrt{E_{0}-k_{\|}^{2}}$ имеет вид

$$
\sqrt{|V|} R(k) \sqrt{V}=-\frac{\sum_{n=1}^{n_{0}}\left(\cdot, \phi_{n}^{*}\right) \phi_{n}}{2 k_{3}^{(0)}\left(k_{3}-k_{3}^{(0)}\right)}+Q(k),
$$

функиия $Q(k)$ аналитически зависит от $k_{3}, \phi_{n}=\sqrt{|V|} \psi_{n}, \phi_{n}^{*}=\sqrt{V} \psi_{n}$, где $\psi_{n} \in$ $L^{2}(\Omega), \quad n=1, \ldots, n_{0},-$ ортонормированный набор собственных функиий оператора $H\left(k_{\|}\right)$, отвечающих собственному значению $E_{0}$.

ДокАЗАТЕЛЬСТво аналогично доказательству теоремы 3 с очевидными изменениями.

ЗАмЕчАниЕ 3 . Таким образом, в случае $n_{0}=2$ формула (33) дает в условиях теоремы 7 обычную формулу теории возмушений. Поправка первого порядка к собственному значению на непрерывном спектре кратности два вешественна.

ЗАмЕчАниЕ 4. Подобно тому, как это сделано в теоремах 1,2 для поправок первого порядка, из соотношений (19), (21) и (22) легко получить для невырожденного уровня и соответствуюшего решения уравнения (15) (а также, чуть более сложно, и в случае вырождения) формулы для поправок любого порядка в терминах оператора $Q(k)$.

ЗАмечаниЕ 5 . Результаты разделов $2,3,5$ (кроме теоремы 7 ) без сушественных изменений переносятся на случай оператора $\Delta+V(x)$, действующего в $L^{2}\left(\mathbb{R}^{3}\right)$, где $V(x)-$ вешественный потенциал, удовлетворяющий оценке $|V(x)| \leqslant C e^{-a|x|}, a>0$, возмущение $\epsilon W(x)$ берется такое, что $W / V \in L^{\infty}\left(\mathbb{R}^{3}\right)$, а $k_{3}$ заменяется на $k=\sqrt{E}$. 


\section{Список литературы}

[1] М. Рид, Б. Саймон. Методы современной математической физики. Т. 4. Анализ операторов. М.: Мир, 1982.

[2] E. B. Davies. Proc. Cambr. Philos. Soc. 1977. V. 82. P. 327.

[3] Ю. П. Чубурин. ТМФ. 1997. Т. 110. С. 443.

[4] Ю. П. Чубурин. ТМФ. 1987. Т. 72. С. 120.

[5] J. S. Howland. Pacific J. of Math. 1974. V. 55. № 1. P. 157.

[6] А. И. Базь, Я. Б. Зельдович, А. М. Переломов. Рассеяние, реакции и распады в нерелятивистской квантовой механике. М.: Наука, 1966.

[7] J. Rauch. J. Funct. Anal. 1980. V. 35. P. 304.

[8] Ю. П. Чубурин. ТМФ. 2001. Т. 126. С. 196.

[9] J. S. Howland. J. of Math. Anal. and Appl. 1971. V. 36. P. 12.

[10] S. Albeverio, F. Gesztezy, R. Høegh-Krohn, W. Kirsch. J. Operator Theory. 1984. V. 12. P. 101.

[11] F. Gesztezy. Perturbation theory for resonances in terms of Fredholm determinants. In: Resonances - Models and Phenomena. Proc. of the Workshop (Bielefeld, 1984). Lect. Notes in Phys. Eds. S. Albeverio, L. S. Ferreira, L. Streit. Berlin: Springer, 1984. V. 211. P. 78.

[12] С. Альбеверио, Ф. Гестези, Р. Хоэг-Крон, Х. Хольден. Решаемые модели в квантовой механике. М.: Мир, 1991.

[13] М. С. Сметанина, Ю. П. Чубурин. Вестн. Удм. ун-та. Математика. 2003. С. 19.

[14] М. Рид, Б. Саймон. Современные методы математической физики. Т. 1. Функциональный анализ. М.: Мир, 1977.

[15] Ю. П. Чубурин. ТМФ. 1998. Т. 116. С. 134.

[16] Р. Ганнинг, Х. Росси. Аналитические функции многих комплексных переменных. М.: Мир, 1969.

[17] Х. Цикон, Р. Фрёзе, В. Кири, Б. Саймон. Операторы Шредингера с приложениями к квантовой механике и глобальной геометрии. М.: Мир, 1990.

[18] Ф. Рисс, Б. Секефальви-Надь. Лекции по функциональному анализу. М.: Мир, 1979.

Поступила в редакцию 8.IX.2004 г., после доработки 24.I.2005 г. 